\title{
Transcriptome sequencing reveals altered long intergenic non-coding RNAs in lung cancer
}

\author{
Nicole M White ${ }^{1 \dagger}$, Christopher R Cabanski ${ }^{1,2 \dagger}$, Jessica M Silva-Fisher ${ }^{1,2}$, Ha X Dang ${ }^{1,2}$, Ramaswamy Govindan ${ }^{1,3}$ \\ and Christopher A Maher ${ }^{1,2,3,4^{*}}$
}

\begin{abstract}
Background: Long intergenic non-coding RNAs (IncRNAs) represent an emerging and under-studied class of transcripts that play a significant role in human cancers. Due to the tissue- and cancer-specific expression patterns observed for many IncRNAs it is believed that they could serve as ideal diagnostic biomarkers. However, until each tumor type is examined more closely, many of these IncRNAs will remain elusive.

Results: Here we characterize the IncRNA landscape in lung cancer using publicly available transcriptome sequencing data from a cohort of 567 adenocarcinoma and squamous cell carcinoma tumors. Through this compendium we identify over 3,000 unannotated intergenic transcripts representing novel IncRNAs. Through comparison of both adenocarcinoma and squamous cell carcinomas with matched controls we discover 111 differentially expressed IncRNAs, which we term lung cancer-associated IncRNAs (LCALs). A pan-cancer analysis of 324 additional tumor and adjacent normal pairs enable us to identify a subset of IncRNAs that display enriched expression specific to lung cancer as well as a subset that appear to be broadly deregulated across human cancers. Integration of exome sequencing data reveals that expression levels of many LCALs have significant associations with the mutational status of key oncogenes in lung cancer. Functional validation, using both knockdown and overexpression, shows that the most differentially expressed InCRNA, LCAL1, plays a role in cellular proliferation.
\end{abstract}

Conclusions: Our systematic characterization of publicly available transcriptome data provides the foundation for future efforts to understand the role of LCALs, develop novel biomarkers, and improve knowledge of lung tumor biology.

\section{Background}

Lung cancer is among the leading causes of death worldwide and accounts for greater than 150,000 deaths per year just in the United States, greater than the combination of the next three most common cancers (colon, breast and prostate) [1]. To date, lung cancer research has primarily focused on the deregulation of protein-coding genes to identify oncogenes and tumor suppressors that could serve as diagnostic and therapeutic targets, thereby missing long non-coding RNAs (lncRNAs), which have been shown to play a critical role in tumorigenesis $[2,3]$. Historical focus on protein-coding genes in disease

\footnotetext{
* Correspondence: cmaher@dom.wustl.edu

${ }^{\dagger}$ Equal contributors

'Department of Internal Medicine, Division of Oncology, Washington

University School of Medicine, St Louis, MO 63110, USA

${ }^{2}$ The Genome Institute, Washington University School of Medicine, St Louis, MO 63110, USA

Full list of author information is available at the end of the article
}

pathology is due to the relatively recent discovery of lncRNAs, the bias of previous technologies (such as microarrays) towards protein-coding genes, and the lack of sufficient datasets to identify lncRNAs in lung cancer.

As part of the ENCODE project, the GENCODE consortium manually curated 9,277 human IncRNAs [4]. However, current estimates suggest that protein-coding genes may be outnumbered by lncRNAs, many of which have yet to be discovered due to their tissue-specific expression profiles and lower expression levels than coding genes [4]. The tissue-specific nature of lncRNAs suggests they may serve as valuable clinical markers [4-6]. However, until we examine each tumor type more closely, many of these clinically relevant lncRNAs may remain elusive. Transcriptome sequencing, or RNA-Seq, offers an unbiased approach for annotating expressed transcripts [5], as exemplified by the discovery of approximately 1,800 unannotated lncRNAs in a cohort of 102 
prostate cancer patients, of which 121 were associated with progression [6].

Although originally regarded as transcriptional noise, several well-described examples indicate that lncRNAs may be essential actors in cancer biology, typically facilitating epigenetic gene repression through chromatinmodifying complexes. Examples include the increased expression of HOTAIR in metastatic breast cancer [7], $A N R I L$-induced silencing of p15 in leukemia [8], and MALAT1 association with metastasis in non-small cell lung cancer [9]. In contrast to these well-described examples, however, only a fraction of lncRNAs have documented roles in tumorigenesis [10-12] and even fewer have been implicated in lung cancer. The most well-characterized lncRNA reported in lung cancer is MALAT1 (metastasis-associated lung adenocarcinoma transcript 1), which is associated with high metastatic potential and poor patient prognosis in non-small cell lung cancer patients with and without metastatic tumors $[9,13]$. More recent studies have found that the intronic non-coding RNA (ncRNA) lncRNA-LET plays a role in the regulation of hypoxia-mediated metastasis in squamous cell lung carcinoma [14], intronic ncRNA AK126698 confers resistance to cisplatin by targeting the Wnt pathway [15], and the lncRNA SCAL1 (smoke and cancer-associated $\ln C R N A-1$ ) is associated with tobacco-induced lung cancer [16]. These individual studies demonstrate the growing importance of lncRNAs in lung cancer while highlighting the need to systematically identify lncRNAs altered in lung cancer. Given the vast quantity of lncRNAs detected and still being discovered, this represents a unique research opportunity to uncover novel biomarkers and therapeutic targets, and to understand their role in tumor biology.

In our study we harnessed the unbiased view of the transcriptome offered by massively parallel next-generation sequencing platforms to explore the recently emerging class of lncRNAs in lung cancer from 197 lung squamous cell carcinoma and 370 adenocarcinoma tumors. Overall, we were able to detect over 3,000 previously unannotated lncRNAs and identify 111 lncRNAs, termed lung cancerassociated lncRNAs (LCALs), that are strongly differentially expressed between lung tumors and adjacent normal tissue. For orthogonal validation we repurposed publicly available exon array-based data coupled with experimental validation (quantitative real-time PCR (qPCR) and rapid amplification of cDNA ends (RACE)) for a subset of LCALs. To elucidate the tissue specificity of lncRNAs altered in lung cancer we conducted a meta-analysis across an additional 324 tumor and adjacent normal pairs from seven different cancers that were sequenced as part of The Cancer Genome Atlas (TCGA) project. Additionally, we incorporated exome sequencing data from TCGA to identify LCALs that were associated with commonly mutated genes. The most differentially expressed lncRNA, $L C A L 1$, was functionally validated and determined to regulate cellular proliferation in vitro. In summary, we have systematically characterized lncRNAs that may play a critical role in lung cancer.

\section{Results}

\section{Identification of novel unannotated transcripts}

To comprehensively characterize the lncRNA landscape in lung cancer we analyzed poly-A purified RNA-Seq data from three cohorts: (1) 197 squamous cell carcinomas with 34 matched adjacent normal from TCGA [17] (LUSC cohort); (2) 298 adenocarcinomas with 55 matched adjacent normal from TCGA (LUAD cohort); and (3) 72 adenocarcinomas and adjacent normal pairs from a Korean population [18] (Seo cohort). To identify novel unannotated transcripts, the aligned reads for each sample underwent de novo assembly using Cufflinks [19] and were subsequently merged together into a consensus lung cancer transcriptome (Figure 1A). As none of these data sets utilized stranded library protocols, we were prevented from discriminating any regions in which two independent transcripts overlap. Therefore, we focused solely on intergenic transcripts (as described in Materials and methods). To ensure that transcripts were not previously annotated, the consensus lung transcriptome was compared against a comprehensive gene database comprised of UCSC [20], Ensembl [21], GENCODE [22], and RefSeq [23] as well as a set of lncRNAs in human development [5]. To remove extensions of annotated transcripts, we filtered any transcript intersecting a protein-coding exon. Last, transcripts lacking a splice junction, and therefore could be due to potential DNA contamination, or less than 200 nucleotides in length were filtered. This resulted in the discovery of 3,452 multi-exon genes residing within intergenic regions of the genome (Table S1 in Additional file 1).

\section{Characterization of novel IncRNAs}

To ensure that the novel candidates that we predicted did not encode proteins, we used GeneID [24] and CPAT [25] to measure (1) the protein-coding potential and (2) the ORF size in each lncRNA sequence. For comparison, genes were classified into four categories: (i) unannotated transcripts (Novel); (ii) non-coding RNAs annotated by RefSeq (Known_RNA); (iii) proteincoding genes annotated by RefSeq (mRNA); and (iv) previously annotated lncRNAs (lncRNAs) [5]. The unannotated transcripts have a lower coding potential and ORF length relative to protein-coding genes but similar coding potential to known RNA genes and recently reported lncRNAs (Figure 1B; Figure S1A,B in Additional file 2; Table S2 in Additional file 1). Additionally, the expression levels of the novel unannotated transcripts were skewed towards lower expression, which was also observed with annotated RNAs and recently discovered 


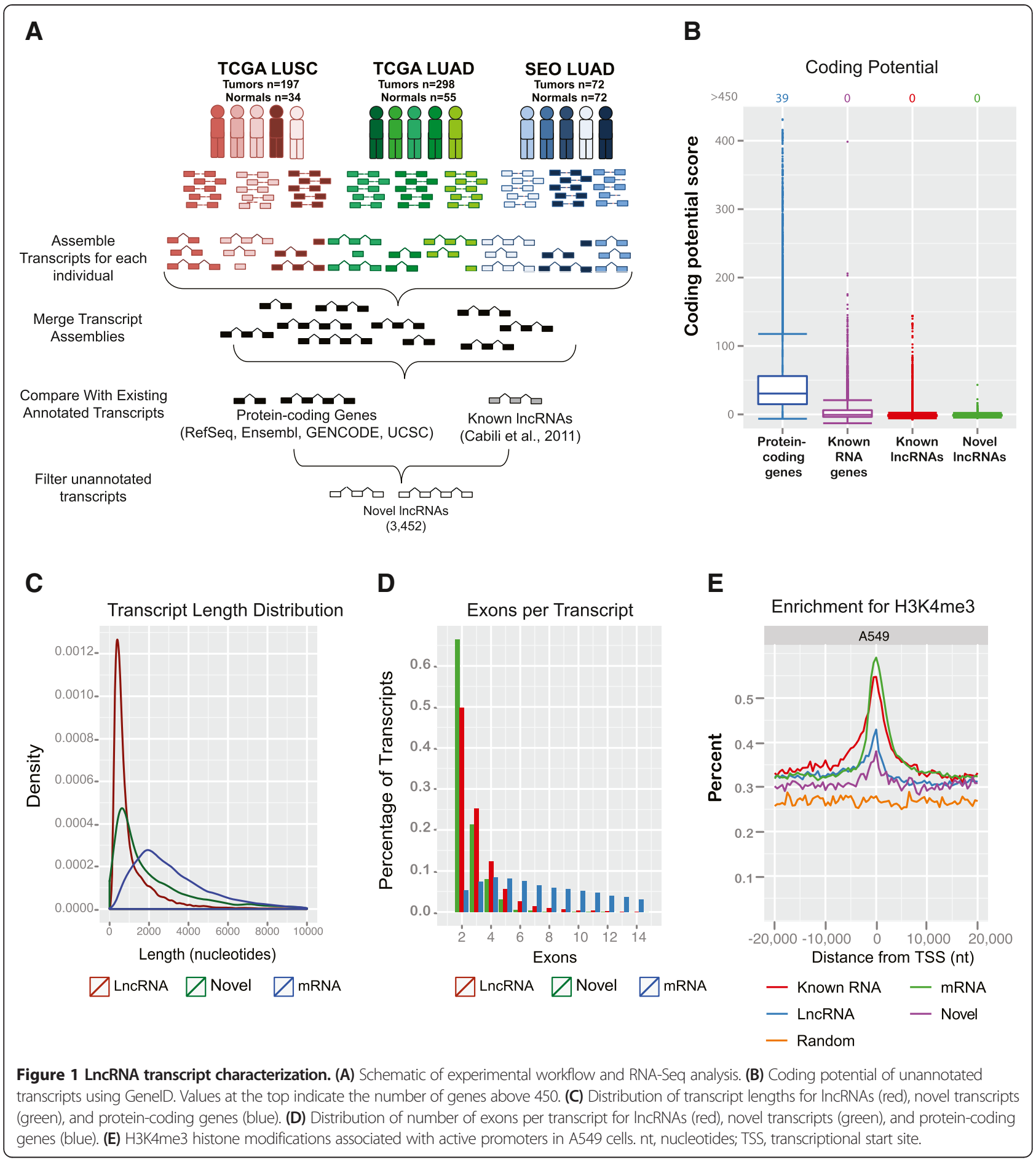

lncRNAs (Figure S1C in Additional file 2). In addition to expression levels, the transcript characteristics of the novel lncRNAs mimic previously reported lncRNAs. As shown in Figure 1C, the overall transcript length of the novel lncRNAs (median 1,823 nucleotides) is shorter than protein-coding genes (2,757 nucleotides; $t$-test, $P$-value $\left.<5.4 \times 10^{-8}\right)$, which is expected given the bias of
IncRNAs having fewer exons than protein-coding genes (Figure 1D).

It was recently found that transposable elements significantly contributed to the origin, diversification, and regulation of lncRNAs in human and vertebrates [26,27]. Consistent with earlier reports [26,27], we also found that repetitive elements accounted for $30.2 \%$ of the novel 
lncRNAs, with the most abundant families including LINE/ L1, LINE/L2, SINE/Alu, SINE/MIR, and LTR/ERVL-MaLR (Figure S1D in Additional file 2).

To determine whether the predicted novel lncRNAs are independent transcripts rather than extensions of neighboring protein-coding transcripts [28], we leveraged existing ENCODE ChIP-Seq data available for the H3K4me3 histone modification that is associated with active promoters. We focused on the epithelial cell line (A549) derived from a lung carcinoma tissue to better reflect our tumor tissue cohort. We observed enrichment for histone modifications characterizing transcriptional start sites and active transcription (Figure 1E). Protein-coding transcripts had the highest enrichment, with recently discovered lncRNAs [5] and novel lncRNAs showing nearly equivalent profiles. Taken together, characterization of the unannotated transcripts suggests that they are novel lncRNAs.

\section{Altered IncRNA expression in lung cancer tissues relative to adjacent normal lung tissues}

An initial investigation of well-characterized lncRNAs across cancers (reviewed in [2,3]) revealed that most lncRNAs with known oncogenic function either do not appear to be altered in our lung cohorts or are very lowly expressed (Figure 2A). For instance, although HOTAIR appears to have a strong log fold change between tumor and normal tissues, its median tumor expression level is <0.1 FPKM (fragments per kilobase of transcript per million mapped reads). Therefore, we sought to identify lncRNAs showing significant expression differences between tumors and normal lung tissues in each of the three cohorts. Before testing for differential expression, we applied a series of filtering steps (see Materials and methods) to focus on intergenic noncoding RNAs displaying reliable expression levels across a majority of the samples (Figure S2A in Additional file 2). We identified 1,027 differentially expressed lncRNAs in LUSC, 592 in LUAD, and 481 in Seo (Tables S3 to S5 in Additional file 1; Figure S2B-D in Additional file 2). Of these, 240 were commonly differentially expressed in all three cohorts (55 up- and 185 downregulated; Figure 2B,C).

Using the results from all three cohorts, we composed a list of 111 intergenic lung cancer-associated lncRNAs (LCALs) that represent the most highly expressed and differentially expressed transcripts (Figure 2D; Table S6 in Additional file 1). Fifty LCALs were differentially expressed in all three cohorts, 22 in two cohorts, and 39 unique to a single cohort. Not surprisingly LUSC had the most cohort-specific lncRNAs, as it is the only squamous cell lung cancer cohort in the study. Additionally, 57 LCALs were differentially expressed in both adenocarcinoma cohorts and 21 were differentially expressed in a single adenocarcinoma cohort. The differences between the LUAD and Seo lncRNAs may represent differences in the ethnic backgrounds amongst the patient population since the Seo cohort is an exclusively Korean patient population.

The 111 LCALs include a lncRNA known to play a role in lung cancer (SCAL1 [16]), cancer-associated lncRNAs not previously implicated in lung cancer (CCAT1 [29], ESCCAL-1 [30], LINC00261 [31], linc-UBC1 [32], UCA1 [33], ENST00000547963 [34], and PART-1 [35]), a lncRNA implicated in a lethal lung developmental disorder (FENDRR [36]), and three previously unannotated lncRNAs. Interestingly, the remaining 99 lncRNAs were previously annotated in normal human tissues but not implicated in human disease.

\section{LncRNAs associated with lung cancer subtypes}

Lung cancer is a heterogeneous disease comprised of different subtypes and molecular aberrations. Therefore, we next sought to better understand the role of lncRNAs in each subtype. We found 463 and 315 up- and downregulated genes, respectively, in LUAD tumors relative to LUSC (Table S7 in Additional file 1; Figure S3 in Additional file 2). Of the 50 LCALs that differentiated tumor from normal tissues across all three cohorts, 27 were differentially expressed between LUAD and LUSC tumors. This subset of LCALs could potentially serve as important biomarkers for lung cancer due to their differential expression between tumor and normal lung tissue as well as between adenocarcinoma and squamous cell carcinoma tumors.

\section{Orthogonal validation of altered lung adenocarcinoma IncRNAs using Affymetrix exon arrays}

To provide additional independent validation of altered lncRNA expression, we repurposed the existing Affymetrix Human Exon 1.0 ST array with publicly available expression profiling data from an independent cohort of 20 adenocarcinoma lung cancer patient tumor and adjacent normal samples collected at the University of Pittsburgh (Gene Expression Omnibus accession GSE12236) [37]. In total, $81.25 \%$ of all lncRNAs were covered by at least one probeset overlapping an exon (including $57.9 \%$ of the 3,246 novel lncRNAs). Of the 111 LCALs, 98 (88.3\%) were covered by at least one probeset. This demonstrates that although the Human Exon Array is able to measure expression levels of a large number of lncRNAs, it does not provide the same genome-wide coverage as RNASeq and therefore misses potentially informative lncRNAs.

Next, we wanted to determine whether the LCALs were also differentially expressed in the adenocarcinoma array data. We restricted our analysis to 66 LCALs that were covered by at least one probeset and differentially expressed in at least one of the adenocarcinoma cohorts. 


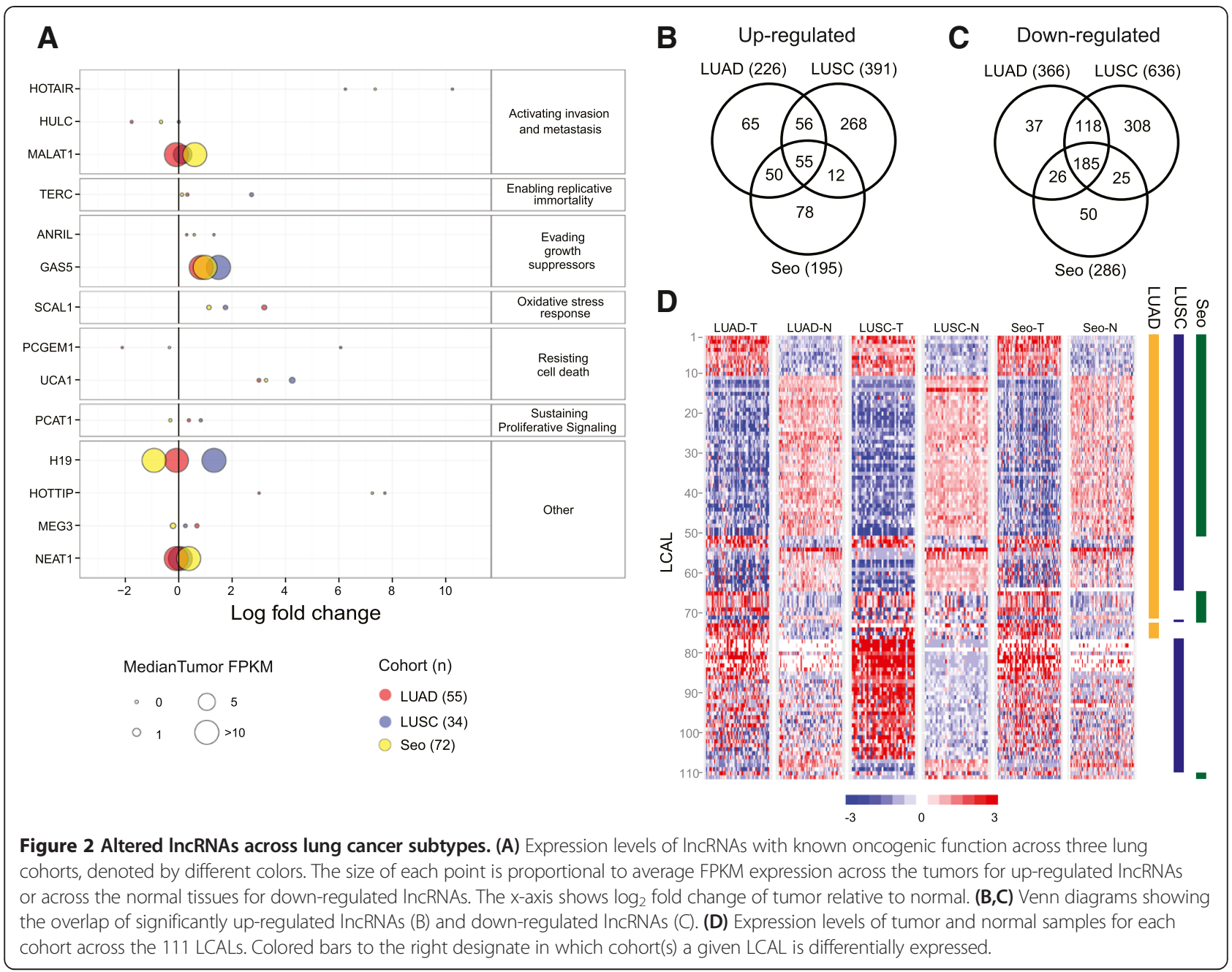

The array confirmed differential expression of 45 of the $66(68.2 \%)$ LCALs (Figure 3; Table S8 in Additional file 1). This validation rate increased to $83.3 \%(40 / 48)$ when considering LCALs called differentially expressed in both adenocarcinoma cohorts.

\section{Experimental validation of LCALs in cell lines and an independent tissue panel}

To further confirm alterations of lncRNA expression in lung cancer, we validated a subset of LCALs across a panel of lung cancer cell lines by qPCR (Figure S4 in Additional file 2). Moreover, we confirmed the cancer-specific expression of the six lncRNAs by qPCR in an independent cohort of lung tissues, collected at Washington University, comprised of adenocarcinoma with matched control tissue and squamous cell carcinoma and matched control tissue (Figure 4; Figure S5 in Additional file 2). This independent cohort confirmed the subtype-specific expression of $L C A L 80$ and LCAL85 (Figure S5E,F in Additional file 2).
LncRNAs are known to display features typical of transcription by RNA polymerase II, including $5^{\prime}$ capping, 3' polyadenylation, and intron splicing [38]. However, despite observing H3K4me3 marks, indicative of promoter regions for the novel lncRNAs, we were still concerned that the lower expression levels of lncRNAs would poorly define the transcript boundaries. Therefore, to characterize the lncRNA transcripts and ensure that we observe the full-length transcript, we designed gene-specific primers for four lncRNA genes and conducted 5' RACE and 3' RACE using Invitrogen's Gene Racer Kit. In each instance we were able to recapitulate a full-length transcript corresponding to the observed RNASeq coverage (Figure 4; Figure S5 in Additional file 2).

\section{Aberrantly expressed IncRNAs across human cancers}

We next investigated whether the identified LCALs have tissue-specific expression profiles, ideal for a putative biomarker, or are altered across numerous human cancers, suggesting that they may have a more common oncogenic or tumor suppressive role in multiple cancers. 


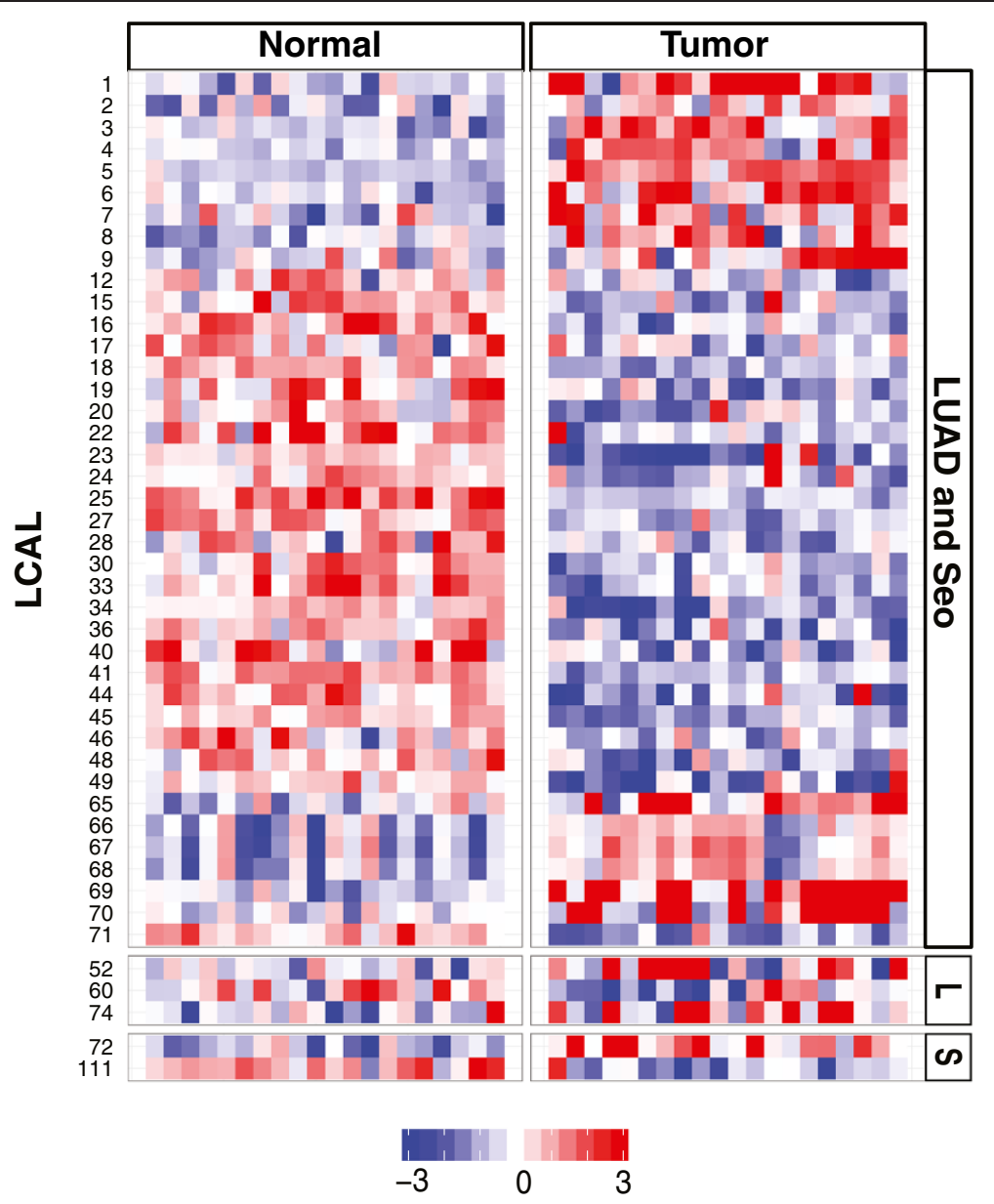

Figure 3 Independent validation of lung cancer-associated IncRNAs. Heatmap of 45 LCALs confirmed as differentially expressed in lung adenocarcinoma tumor and matched normal tissues by Human Exon Array. LCALs are grouped by the RNA-Seq cohort in which they were called significant: LUAD and Seo, LUAD only (denoted 'L'), and Seo only (denoted 'S').

We conducted a pan-cancer analysis of RNA-Seq data from 324 matched tumor and adjacent normal pairs from seven additional TCGA solid tumor types (breast invasive carcinoma [39], colon adenocarcinoma [40], head and neck squamous cell carcinoma, kidney renal clear cell carcinoma [41], stomach adenocarcinoma, thyroid carcinoma, and uterine corpus endometrial carcinoma [42]). We found that $52.3 \%$ (58/111) of LCALs were specific to lung cancer and $24.3 \%$ (27/111) were differentially expressed in only one additional cancer type (Figure $5 \mathrm{~A}, \mathrm{~B}$ ). This demonstrates that most LCALs are specific to lung cancer and thus may have potential use as tissue-specific biomarkers.

We further investigated LCALs that were altered across multiple cancers. Of the nine LCALs that were altered in at least three additional cancers, only LCAL84 has been previously studied in cancer. LCAL84 (ENST00000547963) is a member of a three-lncRNA signature associated with the survival of patients with esophageal squamous cell cancer [34]; thus, it is not unexpected that it is differentially expressed in the two squamous cell cohorts, head and neck and lung, although it is also differentially expressed in colon and stomach adenocarcinoma. Two of the experimentally validated LCALs, LCAL5 and LCAL80, were also broadly altered across three additional cancers (Figure $5 \mathrm{C}$ ). This meta-analysis emphasizes the potential significance of previously uncharacterized lncRNAs across multiple cancers.

\section{Associations with mutation status}

A recent study demonstrated the impact of oncogeneactivating mutations on lncRNAs [43]. Therefore, to determine if LCAL expression levels are associated with mutational status we focused on 16 protein coding genes that have been reported by TCGA as mutated in at least $10 \%$ of lung cancer tumors [44]. We tested each TCGA lung cohort separately due to differences in the mutational frequencies between the subtypes. In LUAD, TP53 and KEAP1 mutational status are associated with 19 and 8 LCALs, respectively. In LUSC, NFE2L2 mutational status is associated with six LCALs (Figure 6A). None of 

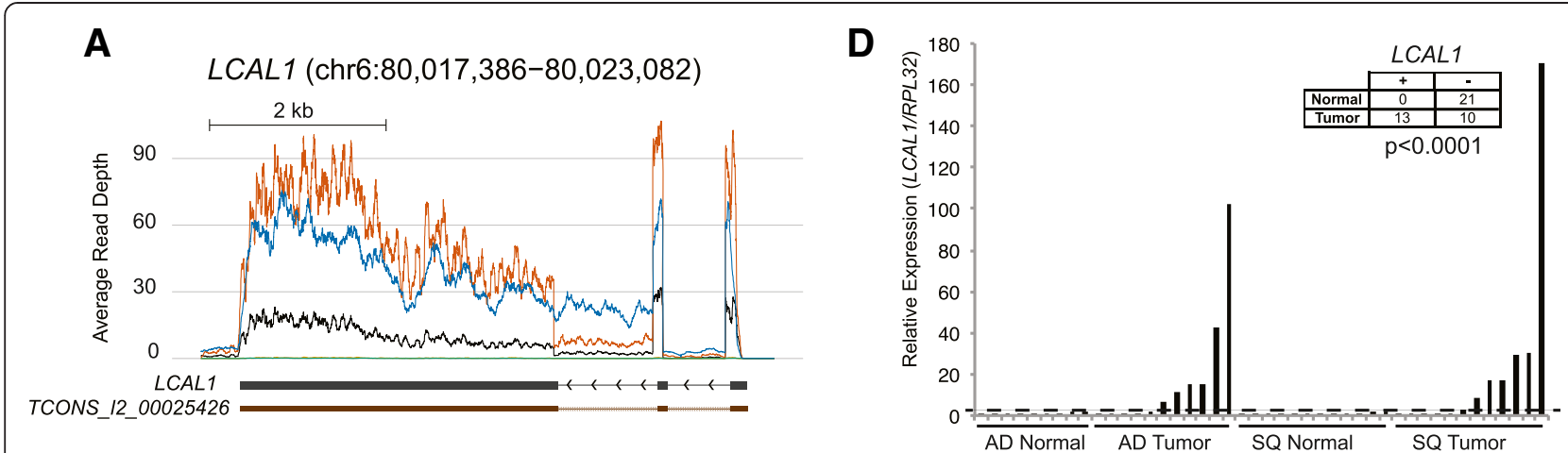

B

LCAL5 (chr17:70,399,468-70,588,876)

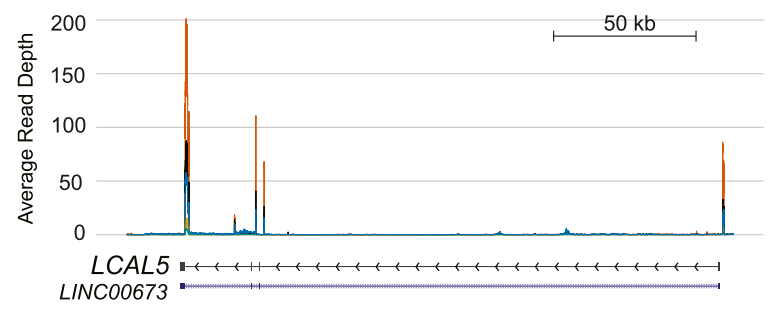

C

LCAL7 (chr8:144,823,553-144,828,510)

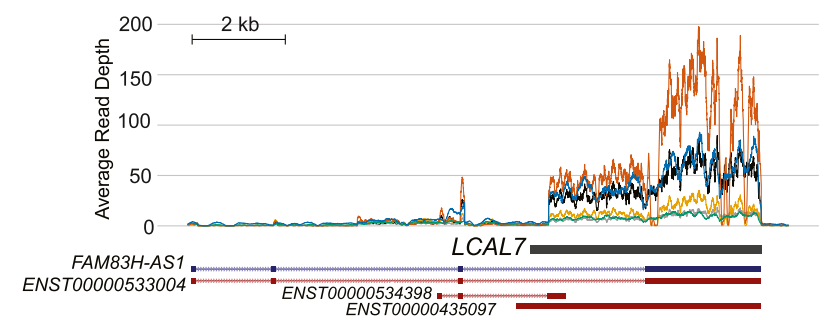

$$
- \text { LUADN }- \text { LUSCN }- \text { SeoN }
$$$$
\text { - LUADT }- \text { LUSC T - SeoT }
$$

E

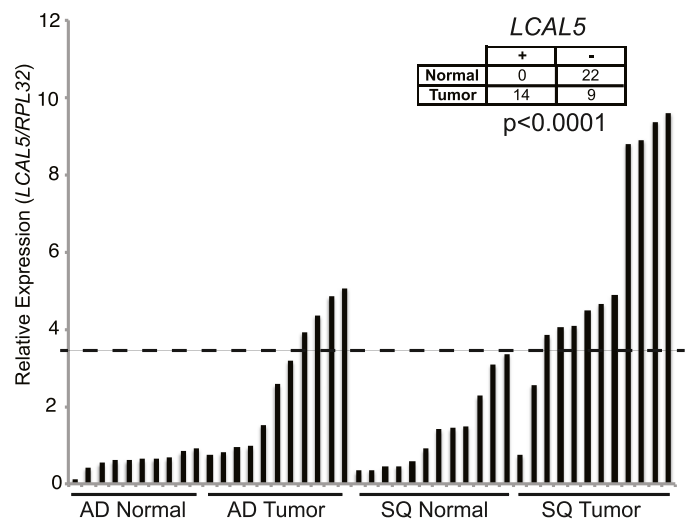

$\mathbf{F}$

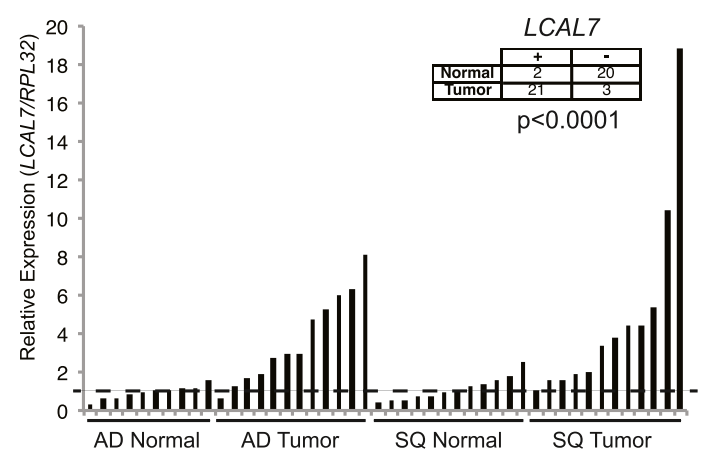

Figure 4 LCAL expression in lung cancer. (A-C) Coverage maps showing the average expression levels of tumor and normal samples across all three lung cancer cohorts for LCAL1 (A), LCAL5 (B), and LCAL7 (C). Annotated RefSeq (dark blue), Ensembl (red), Human Body Map IncRNAs (brown), and full-length transcripts as determined by $5^{\prime}$ and $3^{\prime}$ RACE in H322M cell line (black) are shown below each plot. (D-F) qPCR validation in an independent cohort of human adenocarcinoma and matched controls and squamous cell carcinoma and matched controls for LCAL1 (D), LCAL5 (E), and LCAL7 (F). Insert tables distinguish 'high' and 'low' expression of LCALs in tumors using the value as denoted by the dotted line.

the remaining mutations in either LUAD or LUSC had an association with more than a single LCAL. The mutational status of NFE2L2 and KEAP1, which have been shown to regulate cell response to oxidative damage [45], is associated with expression levels of multiple LCALs, including LCAL51, or SCAL1 (Figure 6B). Additional significant associations with TP53, NFE2L2/KEAP1, CDKN2A, and HGF are shown in Figure S6 (Figure S6 in Additional file 2).

\section{Characterization of LCAL1}

To determine if the lncRNAs found in this study have phenotypic consequences, we chose to examine the most differentially expressed lncRNA, LCAL1, in both adenocarcinoma and squamous cell carcinoma. LCAL1 is located on chromosome 6q14.1 and produces a three-exon transcript (Figure 4A). ENCODE data show DNasel hypersensitivity and transcription factor binding upstream of 


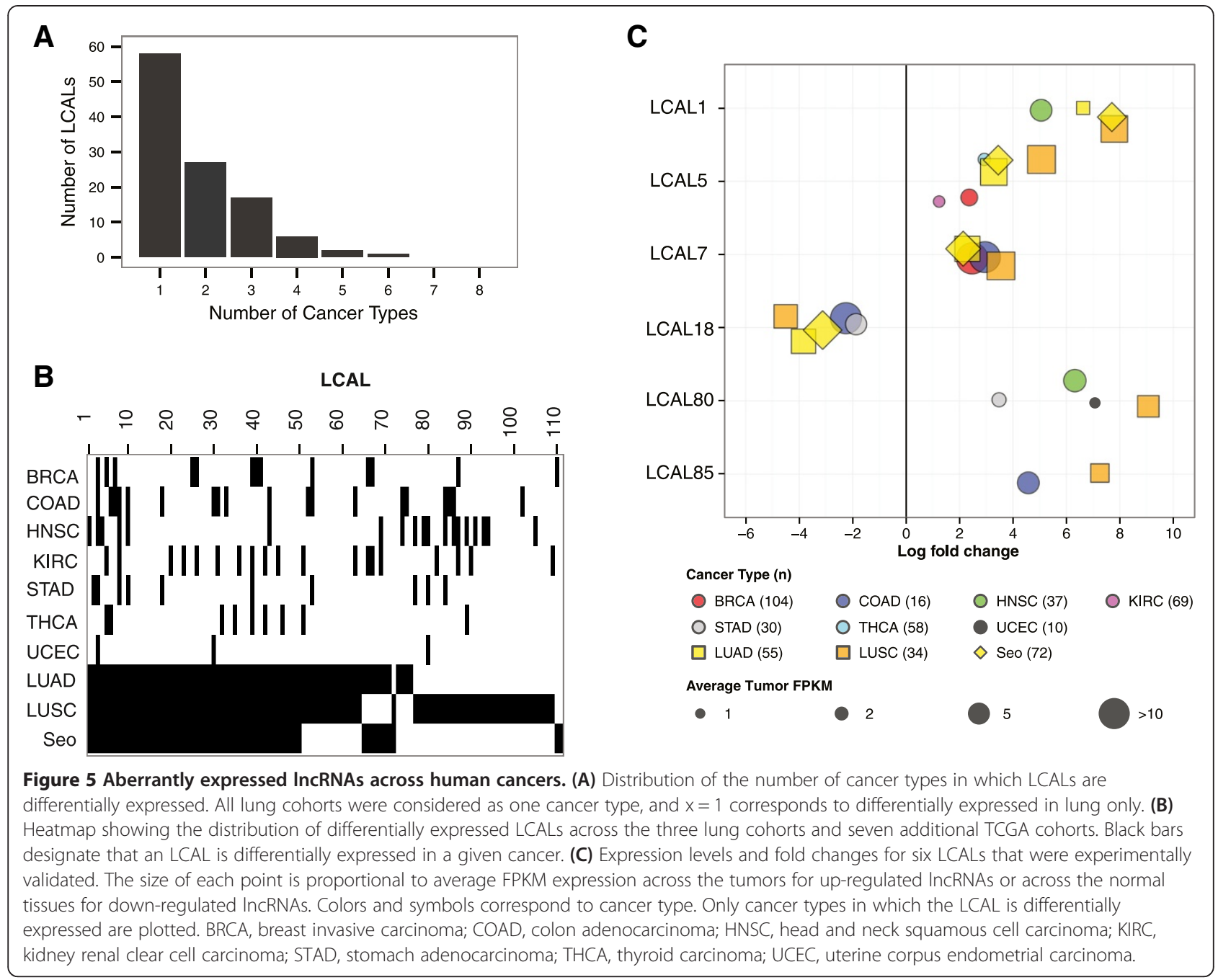

$L C A L 1$, suggesting regulatory activity within the $L C A L 1$ promoter (Figure S7 in Additional file 2). Interestingly, LCAL1 lacks strong base pair conservation, using PhyloP. However, LCAL1 appears to be evolutionarily conserved amongst primates, suggesting a more recent evolution (Figure S7 in Additional file 2). Subcellular localization revealed that LCAL1 was enriched in the nucleus, which is common amongst lncRNAs associated with gene regulation [4] (Figure S8 in Additional file 2).

Next, we wanted to assess the functional significance of LCAL1. Short interfering RNAs (siRNAs) were designed to help assess the function of $L C A L 1$ in lung cancer. Greater than $50 \%$ knockdown of $L C A L 1$ in the cell line H322M, which models adenocarcinoma, with two different siRNAs resulted in decreased cell growth as measured by cell counting for six days. Both LCAL1 siRNA knockdowns in H322M caused at least a $24 \%$ decrease in cell growth starting at day 2 and a $37 \%$ or $50 \%$ decrease in cell growth in siRNA 1 or siRNA 2, respectively, at day 6 compared to control cells (Figure 7A). In our original panel of nine different cancer cell lines, $L C A L 1$ was only highly differentially expressed in one cell line; therefore, we screened additional squamous cell carcinoma lines and found LCAL1 to be highly differentially expressed in HCC95 (Figure S9 in Additional file 2). Greater than 50\% knockdown of LCAL1 in HCC95 cells recapitulated cell growth observations in the H322M cell. Both siRNA knockdowns in HCC95 caused at least a 30\% decrease in cell growth starting at day 2 , which was maintained through to the end of the experiment at day $6 \mathrm{com}$ pared with control cells (Figure 7B). Furthermore, stable overexpression of $L C A L 1$, using two different clones, in the control cell line BEAS-2B showed a significant increase in cellular proliferation starting on day 2 and continuing until the end of the experiment at day 6 with a $38 \%$ and $43 \%$ growth increase, respectively (Figure 7C). Overexpression of LCAL1 in normal BEAS-2B cells, at physiological levels in human tumors, is proof of principle that this lncRNA is sufficient to affect cellular growth independently of other 


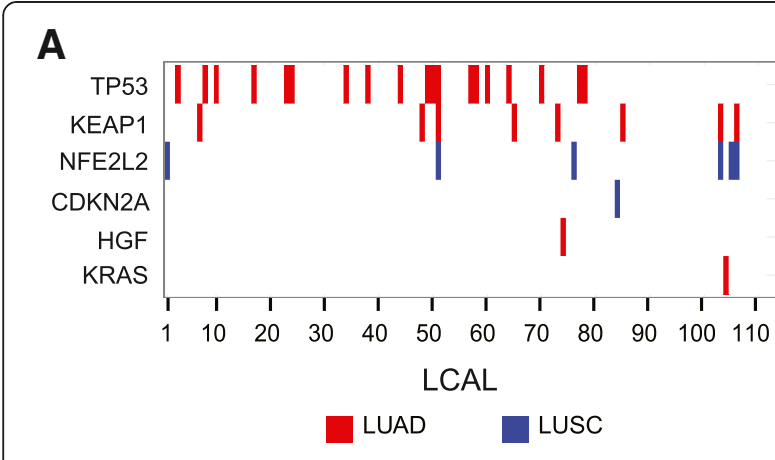

B

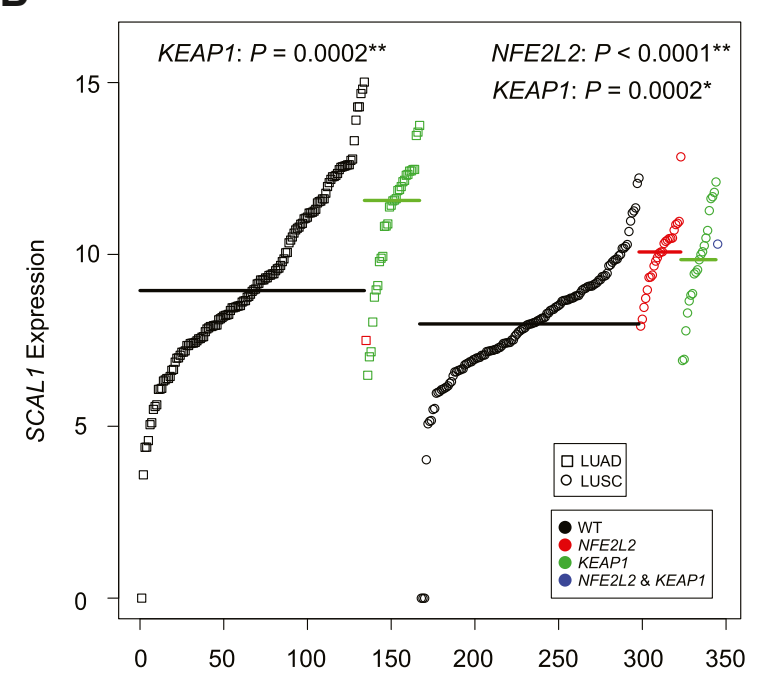

Figure 6 Association between LCAL expression and mutation status. (A) Significant associations between LCAL expression and mutation status. Red bars designate significant associations (false discovery rate (FDR) $<0.01$ ) across LUAD tumors and blue bars across LUSC tumors. (B) Expression levels of SCAL1 (LCAL51), measured by $\log _{2}$ FPKM, for wild-type (WT; black), NFE2L2 mutant (red), KEAP1 mutant (green), and both NFE2L2 and KEAP1 mutant (blue) samples. Data points are ordered by expression levels and symbols designate cohort (squares for LUAD, circles for LUSC). Thick colored lines represent the median expression levels across each group. $P$-values for each mutational association are also reported ( ${ }^{*} \mathrm{FDR}<0.05$, ${ }^{* *} \mathrm{FDR}<0.01$ ).

common cancer mutations, thus highlighting the importance of $L C A L 1$ in lung cancer biology.

To confirm that changes in cell growth were due to a proliferative effect of LCAL1 expression, Alamar Blue proliferation experiments were also conducted. After 72 hours LCAL1 knockdown cells were replated and Alamar Blue reduction was assessed on days 2, 4, and 6 . We see a similar significant decrease of proliferation in both siRNA constructs compared with scrambled control in both H322M and HCC95 cell lines (Figure S10 in Additional file 2). In addition, there was no change in apoptosis or necrosis in both cell lines with decreased $L C A L 1$ expression compared with control as measured by annexin $\mathrm{V}$ and propidium iodide staining at 72 hours post-knockdown (data not shown). These results highlight the biological importance of LCAL1 in promoting tumorigenesis.

\section{Discussion}

The utilization of lncRNAs as biomarkers, and more recently active tumorigenic factors influencing protein function, demonstrates the necessity for more extensive studies characterizing and understanding the role of IncRNAs in disease progression. In this study we used an unbiased approach to systematically categorize lncRNAs in 567 tumors from three separate publicly available RNA-Seq lung data sets. We identified 111 intergenic lung cancerassociated IncRNAs, or LCALs, most of which were not previously implicated in cancer development and progression. Further stratification of the 111 LCALs determined 27 LCALs to be subtype-specific, and therefore might serve as important biomarkers to form a molecular signature in stratifying adenocarcinoma and squamous cell carcinoma. A meta-analysis across seven additional cancers established that most (over 50\%) LCALs appear to have restricted expression in lung cancer, suggesting they may be involved in disease pathogenesis and serve as putative biomarkers. Moreover, a small percentage of LCALs are highly differentially expressed in at least one other cancer, with nine being expressed in at least three additional cancers. This analysis highlights the importance of lncRNAs not only in lung cancer but also as broad oncogenic factors and lays the groundwork for future studies to determine the mechanisms by which these newly discovered non-coding RNAs act in cancer progression.

In our study we provided a comprehensive analysis to detect novel lncRNAs across lung cancer patients that led to the annotation of over 3,000 novel lncRNAs. However, to ensure that we were annotating high-confidence candidates we focused on multi-exon genes. Additionally, the publicly available data collections used for this study did not utilize stranded libraries and therefore did not allow for accurate annotation of antisense non-coding RNAs. Furthermore, the data used in this study focused on polyA+RNA and therefore may have missed some non-coding RNAs. However, for the first time we were able to identify solid tumorassociated lncRNAs not previously implicated in lung cancer as well as uncharacterized lncRNAs altered in lung cancer. For example, linc-UBC1 (LCAL6) was discovered in bladder cancer [32]; UCA1 (LCAL52) in bladder [33], ovarian [46] and breast cancer [47]; LINC00261 (LCAL62) in gastric cancer [31]; ESCCAL-1 (LCAL80) [30] and ENST00000547963 (LCAL84) [34] in esophageal squamous cell carcinoma; CCAT1 (LCAL85) in colon cancer [29]; and PART1 (LCAL92) in prostate cancer [35] and glioblastoma multiforme [48]. Overall, these findings emphasize the importance of unbiased sequencing approaches to better understand the non-coding RNA landscape of cancer. 

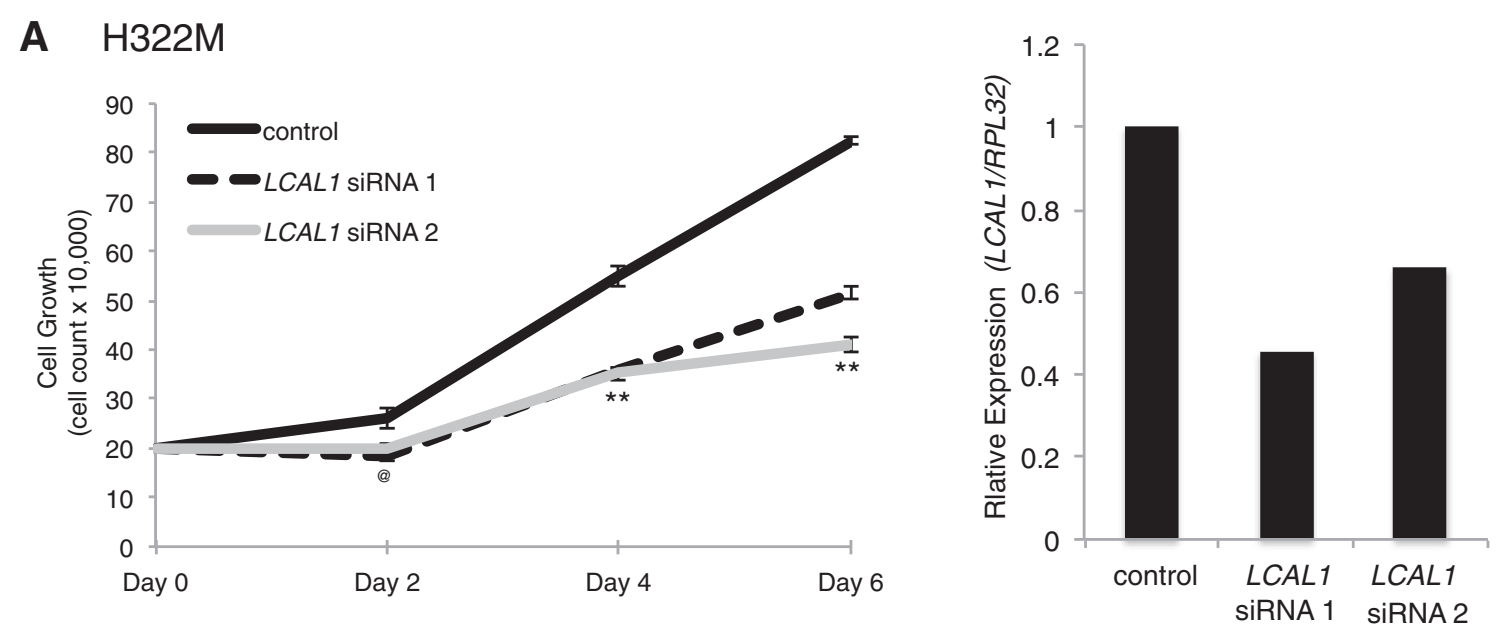

\section{B $\mathrm{HCC} 95$}
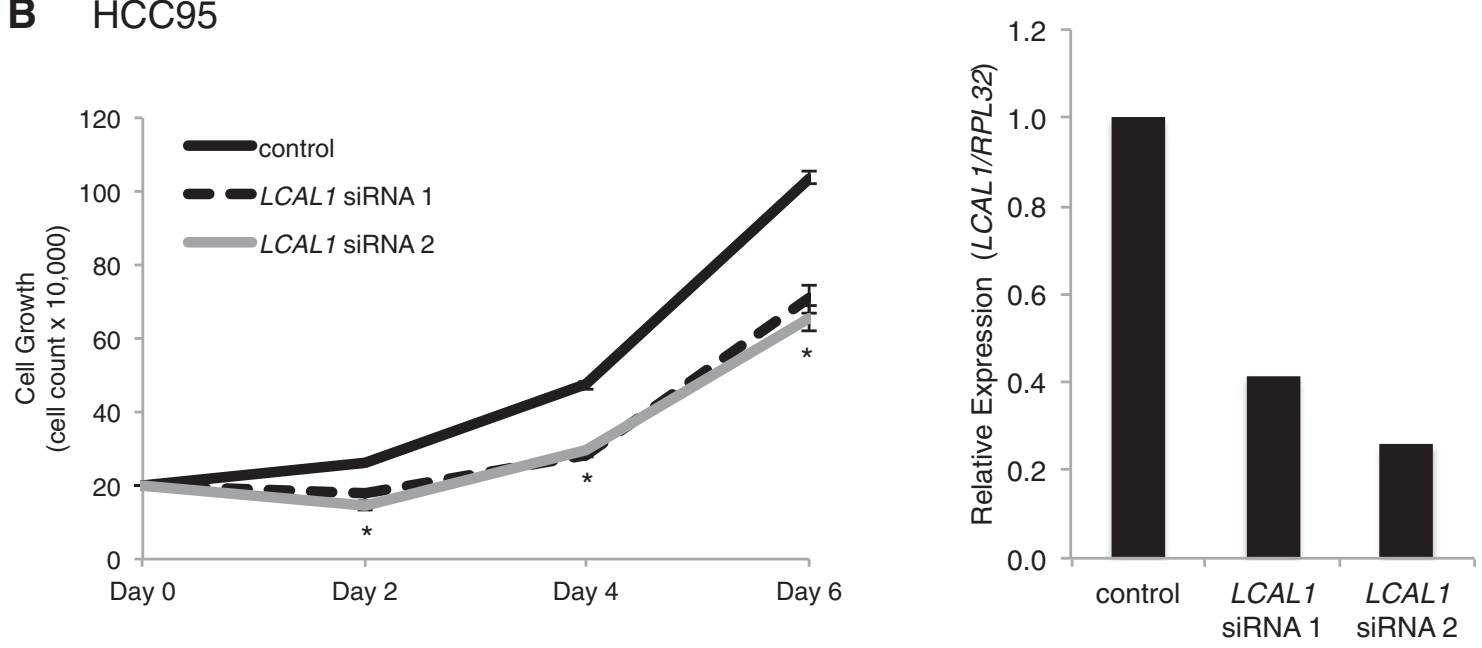

C Beas $L C A L 1$ overexpressing cells
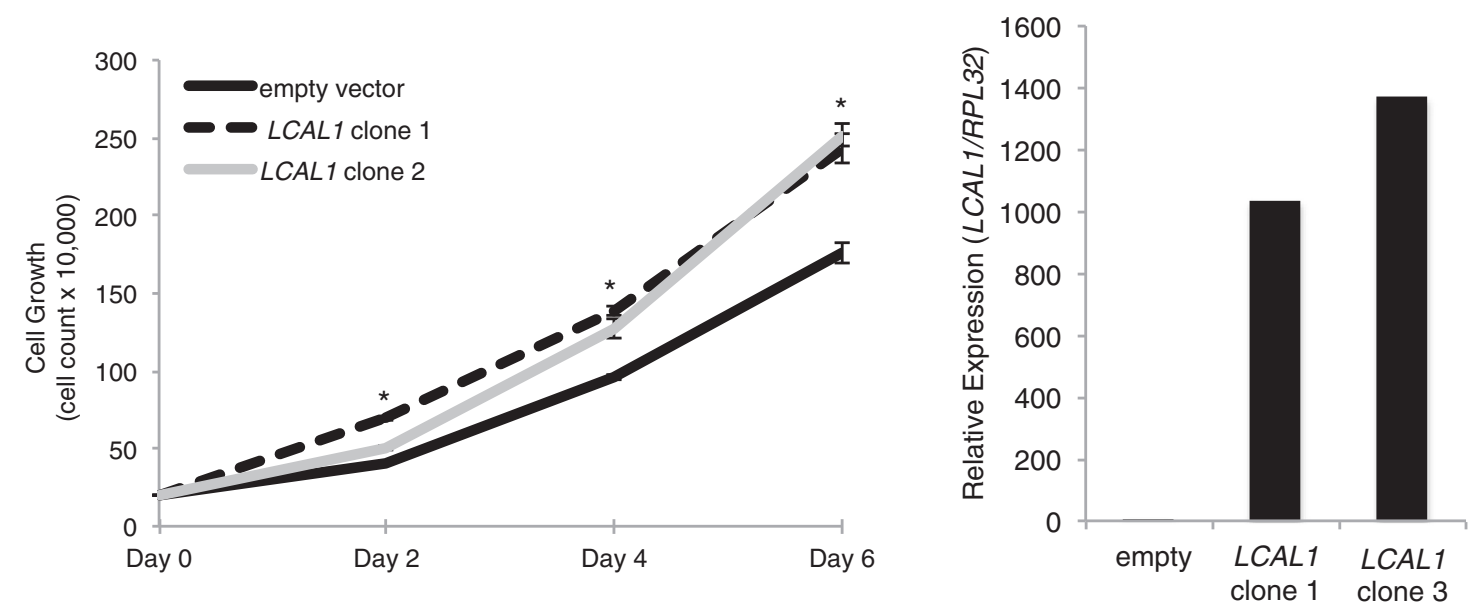

Figure 7 (See legend on next page.) 
(See figure on previous page.)

Figure 7 LCAL1 expression affects cell growth. (A, B) Cell proliferation assay in H322M (A) and HCC95 (B) cells using LCAL1 siRNAs. qPCR validation of $L C A L 1$ siRNA knockdown is shown on the right. (C) Cell proliferation assay in BEAS-2B overexpressing clones of $L C A L 1$ with $q P C R$ validation of $L C A L 1$ expression in BEAS-2B cells on the right. ${ }^{@} P \leq 0.05,{ }^{*} P \leq 0.01,{ }^{* *} P \leq 0.001$ by a two-tailed Student's $t$-test. The same significance applies for siRNA 1 and siRNA 2 at all time points. All error bars are mean \pm standard error of the mean across $n=3$ biological replicates in two independent experiments.

One of the major challenges for studying lncRNAs is to determine their potential functional role. Interestingly, mutations in well-established oncogenes have shown association with lncRNAs. For example, the lncRNA BANCR was found as a recurrently overexpressed transcript in $B R A F^{\mathrm{V} 600 \mathrm{E}}$-mutant human melanoma, which is the most activating mutation in melanoma, with a potential role in regulating cell migration [43]. Additional studies have

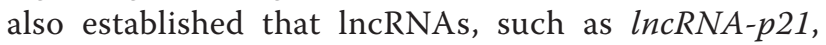
contain functional p53-binding motifs [49], indicating these lncRNAs serve as transcriptional targets in key biological pathways. Here we discovered that some LCALs are also associated with mutational status, thereby implicating them in key oncogenic pathways. In addition to altered LCAL expression associating with TP53 mutation status, some of the LCALs also associated with mutations in KEAP1 and NFE2L2, which are key players in the oxidative stress pathway. For instance, SCAL1 (LCAL51) was found to be associated with KEAP1 mutation status in LUAD and NFE2L2 mutation status in LUSC (Figure 6B) and was recently shown to act downstream of NRF2 and mediate oxidative stress protection in airway epithelial cells [16]. The association of SCAL1 with oxidative stress, which has been previously explored through experimental validation [16], further supports that the association of LCAL expression with mutational status can potentially elucidate their function and serve as the basis for future cancer biology studies.

To determine the importance of these LCALs in lung disease pathology, we proceeded with functional studies of LCAL1, the top up-regulated lncRNA in both LUAD and LUSC. Cellular proliferation studies revealed an oncogenic phenotype, as shown by siRNA knockdown studies of LCAL1 resulting in decreased cellular growth in two cellular models of lung cancer, a non-small cell lung carcinoma cell line (H322M) and a squamous cell carcinoma cell line (HCC95). Moreover, as proof-of-principle, our LCAL1 overexpression studies highlight increased proliferation compared with control BEAS-2B empty vector cells, suggesting that altered $L C A L 1$ is sufficient for promoting the etiology of the disease. Furthermore, our $L C A L 1$ experiments highlight the potential functional contribution additional LCALs may have in various facets of lung tumorigenesis.

\section{Conclusions}

To date, lung cancer research has primarily focused on the deregulation of protein-coding and microRNA genes to identify oncogenes and tumor suppressors as potential diagnostic and therapeutic targets. However, lncRNAs represent an emerging and under-studied class of transcripts that have a significant role in human cancers. This study leverages RNA-Seq data from approximately 550 patient specimens representing an unmatched lung cancer transcriptome analysis to date to discover 111 lung cancerassociated lncRNAs (LCALs). We have experimentally validated a subset of LCALs and demonstrated that the most commonly up-regulated lncRNA across lung subtypes, $L C A L 1$, contributes to cellular proliferation. A metaanalysis across human cancers revealed a subset of LCALs that have restricted expression and may represent putative biomarkers while a subset appear to be altered in multiple solid tumors, suggesting a common oncogenic role. Taken together, our study highlights the comprehensive scope of lncRNAs (both previously known and novel) that may contribute to lung cancer. While we already demonstrate the biological significance of $L C A L 1$, our study provides a framework for subsequent research exploring additional LCALs in lung tumorigenesis as well as assessing their prognostic and predictive potential.

\section{Materials and methods Lung RNA-Seq datasets}

Raw sequences from three previously sequenced lung RNA-Seq datasets were downloaded: (1) 72 adenocarcinoma tumor and adjacent normal pairs [18] (referred to as 'Seo') from EBI-SRA under accession number ERP001058; (2) 55 adenocarcinaoma tumors and adjacent normal pairs, plus an additional 243 unmatched tumors, from TCGA (referred to as 'LUAD'); and (3) 34 squamous cell carcinoma tumors and adjacent normal pairs, plus an additional 163 unmatched tumors, from TCGA [17] (referred to as 'LUSC'). Sequence reads were aligned using TopHat v1.3.0 [50].

\section{Discovery of unannotated IncRNAs}

All available samples (adjacent normal, matched tumor, unmatched tumor) from the LUAD, LUSC and Seo cohorts were used to discover novel expressed transcripts. Transcript assemblies were generated using Cufflinks v2.0.2 [19] in de novo mode and subsequently merged 
together with Cuffmerge to generate a consensus transcriptome across the cohort. To identify unannotated transcripts, a comprehensive set of protein-coding gene annotations was generated by downloading RefSeq, UCSC, Ensembl and GENCODE v17 gene annotations, in gene transfer format (GTF) and aggregated together (each downloaded on 20 September 2013). Additionally, the lncRNAs identified from the Human Body Map project were downloaded from UCSC and aggregated to the protein-coding GTF. Cuffcompare was used to compare the lung cancer consensus transcriptome with our comprehensive proteincoding and lncRNA gene reference. The Cuffcompare results were filtered for gene loci that were classified as unannotated (' $u$ ') and none of the transcripts overlapped an existing gene annotation. This subset was defined as 'novel transcripts'. Analysis of coding potential of lncRNAs was performed on transcript sequences using GeneID [24] and CPAT and were both pre-trained for human genes [25].

Enrichment for H3K4me3 histone modifications in lung cancer cells was conducted using ENCODE ChIPSeq data downloaded from the UCSC browser tracks. Coverage was aggregated across 500 nucleotide bins for $20 \mathrm{~kb}$ upstream and downstream of the transcription start site for each transcript in the following categories: (i) protein-coding, (ii) known RNAs (reported in RefSeq), (iii) IncRNAs recently annotated in human development, (iv) novel lncRNAs found in this study, and (v) random. The random transcriptional start sites were determined by selecting genomic coordinates randomly throughout the genome.

\section{Repetitive element analysis of IncRNAs}

RepeatMasker annotation for human genome (hg19 assembly) was downloaded from the UCSC database [20]. BEDTools intersect [51] was used to identify overlap between repetitive elements and transcript exons. Repetitive elements that overlapped at least 10 nucleotides with an exon were considered for further analysis. Anything that was not classified as a transposable element (such as low complexity, satellites, and simple repeats) was removed from further analysis.

\section{Gene expression analysis}

Figure S2A in Additional file 2 shows the multiple steps of our differential expression pipeline. A custom annotation file comprised of IncRNAs from multiple sources was generated by merging noncoding transcripts from GENCODE v17, Ensembl, UCSC, Human Body Map, and our novel lncRNAs. All single-exon transcripts were removed. This list was then merged with all RefSeq noncoding transcripts, including single-exon transcripts, and all transcripts less than 200 nucleotides were removed. Transcripts overlapping an exon from a RefSeq protein coding gene or Ensembl pseudogene were removed, resulting in 34,308 unique transcripts spanning 14,091 gene loci. Gene expression FPKM values were calculated with Cufflinks v2.0.2 using this custom lncRNA annotation file. Additionally, a table comprising read counts for each transcript was calculated using BEDTools version 2.17.0 [51]. We removed lowly expressed transcripts (at least $75 \%$ of samples had FPKM $<0.1$ or read count $<25$ ). The set of remaining transcripts was reduced to a set of non-overlapping regions (or 'genes') by comparing all overlapping transcripts and keeping the transcript with the largest average FPKM across all samples as the representative transcript for that region. After TMM normalization [52], edgeR version 3.0.8 [53] was used to identify differentially expressed transcripts between tumor and normal pairs using a matched pair design for the Seo, LUAD and LUSC datasets using cutoffs of false discovery rate (FDR) $\leq 10^{-5}$ and absolute fold change $\geq 2$. To obtain the list of LCALs, we selected lncRNAs for which the following criteria all held across at least one cohort: (1) differentially expressed, (2) highly expressed (average tumor or normal FPKM $\geq 2$ ), and (3) large fold change between tumor and normal (fold change $\geq 8$ ).

The same pipeline was used for discovering differentially expressed lncRNAs between tumor subtypes. The only difference was that instead of using a matched pair design, we tested for a difference between subgroups after adjusting for gender (LUAD, $n=297$; LUSC, $n=$ 196; two samples without gender information were removed). Heatmaps were generated for each dataset using standardized values by subtracting the median and dividing by the median absolute deviation of each lncRNA. Rows (lncRNAs) were clustered using Ward's method.

\section{Expression levels of validated LCALs}

For the six LCALs that were experimentally validated (LCALs 1, 5, 7, 18, 80, and 85), coverage across the transcript was calculated by counting the read depth at each base using custom perl scripts and the Bio::DB::Sam BioPerl package. Coverage maps shown in Figure 4 and in Figure S5 in Additional file 2 were created in R using SigFuge version 1.1.2 [54]. For the tables shown in Figure 4 and in Figure S5 in Additional file 2, the cutoff for classifying samples as high or low expression was determined by maximizing the Matthews correlation coefficient [55] and two-sided $P$-values were calculated using Fisher's exact test.

\section{Human exon array validation}

Affymetrix Human Exon 1.0 ST Array data for 20 lung adenocarcinoma tumor and adjacent normal pairs [37] were downloaded from Gene Expression Omnibus (GSE12236). We chose to repurpose this array platform because it has the most comprehensive probe 
coverage of lncRNA genes. The genomic coordinates for each probeset were converted from hg18 to hg19 using the UCSC Genome Browser LiftOver tool [56]. Probesets overlapping exons of lncRNAs were determined using custom perl scripts. For annotated lncRNAs, only probesets on the correct strand were used. For the novel IncRNAs where the strand is unknown, overlapping probes on either strand were used. The raw CEL files were processed using Affymetrix Power Tools [57] with RMA normalization [58] to generate transcript-level intensity estimates. For each LCAL that was called differentially expressed in either adenocarcinoma cohort (LUAD or Seo), a paired Wilcoxon signed rank test was performed. LCALs with $P<0.05$ were considered to be validated by the array.

\section{Aberrantly expressed IncRNAs across human cancers}

TCGA MapSplice aligned BAM files [59] were downloaded from TCGA for tumor and adjacent normal pairs from the following tissue types: breast invasive carcinoma $(n=104$ pairs), colon adenocarcinoma $(n=16)$, head and neck squamous cell carcinoma $(n=37)$, kidney renal clear cell carcinoma $(n=69)$, stomach adenocarcinoma $(n=30)$, thyroid carcinoma $(n=58)$, and uterine corpus endometrial carcinoma $(n=10)$. A table comprising read counts for each transcript from our custom IncRNA annotation file was calculated using BEDTools version 2.17.0 [51]. Similar to the edgeR pipeline previously described, transcripts less than 200 nucleotides, with only one exon, or overlapping known pseudogenes were removed along with protein coding genes. Log fold changes were obtained from edgeR and FPKM [19] expression values were manually calculated as $10^{9}(M /(T \times L))$ where $M$ is the number of reads mapping to a transcript, $T$ is the total number of mapped reads, and $L$ is the transcript length. LncRNAs with FDR $\leq 10^{-5}$, absolute fold change $\geq 2$, altered in the same direction as lung, and either average tumor FPKM or average normal FPKM $\geq 1$ were called significantly differentially expressed.

\section{Association of LCALs with mutation status}

The most frequently mutated genes in lung cancer were determined as having over $10 \%$ mutation rate in either the LUAD or LUSC cohort, as reported in Figure 2 from Kandoth et al. [44]. Mutation calls were downloaded from TCGA [60]. Attention was restricted to 167 LUAD and 178 LUSC samples with both RNA-Seq and mutation data. A Wilcoxon rank sum test was used to test for significance between mutational status and expression of each LCAL (using manually calculated FPKM values). For each mutated gene, $P$-values for the LCALs were corrected for multiple comparisons using the Benjamini and Hockberg FDR correction [61], and a significance threshold of 0.01 was used.

\section{Cell culture and human lung cancer RNA}

A549, HOP62, HOP92, NCI-H522, -H32, -H460, -H322M, and $-\mathrm{H} 226$ were a kind gift from Dr Van Tine at Washington University. Calu-1, SK-MES-1, SW900, and HCC95 were a kind gift from Dr Loren Michel at Washington University. HCC827 was a kind gift from Dr Leonard Maggi at Washington University. BEAS-2B cells were purchased from American Type Culture Collection (Manassas, VA, USA). All cells were grown in RPM1-1640 (Invitrogen, Carsbad, CA, USA) with 10\% fetal bovine serum and $1 \%$ penicillin/streptomycin. RNA $(2 \mu \mathrm{g})$ from lung cancer tissue and their matched controls was obtained from the Tissue Procurement Core at Washington University.

\section{RNA isolation and CDNA synthesis}

Total RNA was isolated with the RNeasy Mini Kit (QIAGEN) with DNase 1 treatment according to the manufacturer's instructions. cDNA was synthesized from total RNA using High Capacity cDNA Reverse Transcription Kit with random hexamers (Invitrogen). Human lung cancer tissue RNA was used to make cDNA with the Superscript III RT-PCR Kit (Invitrogen).

\section{Quantitative real-time PCR}

At least two biological replicates were used for qPCR using PowerSyBr Green (Invitrogen). The comparative CT $(\triangle \Delta C T)$ method was used with values first normalized to the housekeeping gene RPL32, and then to BEAS-2B control. All primers were obtained from Integrated DNA Technologies (Coralville, IA, USA) and are listed in Table S9 in Additional file 1. Primer efficiency between 90 and 110\% was determined for each primer candidate.

\section{RACE}

5' and 3' RACE was done using the GeneRacer Kit (Invitrogen) according to the manufacturer's instructions. RACE PCR products were obtained with Platinum Taq High Fidelity (Invitrogen) using the GeneRacer primer (supplied) and a gene-specific primer (GSP) listed in Table S10 in Additional file 1. Nested PCR was also performed for most transcripts. Products were visualized on a $2 \%$ agarose gel and purified by gel extraction (QIAGEN). This product was then cloned into pcr4-TOPO vector (Invitrogen) and grown in TOP10 Escherichia coli. Clones were sequenced with the M13 forward primer at The Protein and Nucleic Acid Chemistry Laboratory at Washington University. Full-length sequences were uploaded to GenBank under the following accession numbers: KF773845 (LCAL1), KF773846 (LCAL5), KF773847 (LCAL7), and KF773848 (LCAL80). 


\section{siRNA knockdown experiments}

Stealth siRNA oligonucleotides were synthesized by Invitrogen. The following siRNA sequences were used for knockdown of LCAL1: siRNA 1 GGACAGGCTGCAGT CATCATATGGA and siRNA 2 GGCATGTGTTCAGA CATATCCTAAA. Cells were transfected with $50 \mathrm{pmol}$ of siRNA and a scrambled-matched \%GC oligo as control with RNAimax Lipofecatmine (Invitrogen) following the manufacturer's instructions. Knockdown efficiency was determined by qPCR at time of plating for assay. After 72 hours, cells were then plated at 200,000 cells/ well for proliferation assays. Cells were counted using the Beckman Z1 Coulter Counter at days 2, 4, and 6. At least three biological replicates were performed for each siRNA construct over two experiments.

\section{Alamar Blue proliferation assays}

Seventy-two hours after transfection, cells were seeded at 3,000 cells/well in a 96-well dish to assess viability via Alamar Blue according to the manufacturer's instructions (Sigma). Subsequent cells were then used for RNA isolation to detect relative expression of $L C A L 1$. Fluorescence intensity was then measured with Gen 5 software on Synergy Hybrid (BioTek) at days 2, 4, and 6 postknockdown after one hour incubation with Alamar Blue. At least four biological replicates were done for each siRNA construct over two experiments.

\section{Retroviral infection and generation of BEAS-2B cell lines stably expressing $L C A L 1$ variants}

The full length $L C A L 1$ transcript was PCR amplified from H322M cells and cloned into the pCFG5-IEGZ vector (a kind gift from Dr Ron Bose). Full-length inserts were confirmed with Sanger sequencing at The Protein and Nucleic Acid Chemistry Laboratory at Washington University. Retroviral infection of BEAS-2B cells were performed according to Kavuri et al. [62]. Briefly, the amphotrophic producer cell lines were transfected with $10 \mu \mathrm{g}$ of LCAL1 and empty control retroviral vectors by calcium phosphate precipitation and incubated for 24 hours. Viral supernatants were harvested after an additional 24 hour incubation. Virus was added to BEAS-2B cells seeded in six-well dishes in the presence of $8 \mu \mathrm{g} / \mathrm{ml}$ Polybrene. BEAS-2B cells were centrifuged at 2,500 RPM for 1.5 hours at $22^{\circ} \mathrm{C}$ and supernatant exchanged for fresh media. After 10 to 14 days of $125 \mu \mathrm{g} / \mathrm{ml}$ zeocin selection, cells were plated at 200,000 cells/well for proliferation assays. Cells were counted using the Beckman Z1 Coulter Counter at days 2, 4, and 6. At least three biological replicates were performed for each stable cell line over two experiments. Cells were also collected for validation of $L C A L 1$ expression by $\mathrm{qPCR}$.

\section{Nuclear localization}

H322M lysates were fractionated into nuclear and cytosolic fractions according to the PARIS kit protocol (Invitrogen) and gene expression was assessed by qPCR. Results were normalized to the housekeeping gene RPL32, and then to total RNA. U6 was used as a positive control for nuclear gene expression and GAPDH and MT-RNR1 were used as positive cytoplasmic gene expression. Three biological replicates were conducted over two independent experiments.

\section{Additional files}

Additional file 1: Supplementary Tables S1 to S10.

Additional file 2: Supplementary Figures S1 to S10.

\section{Abbreviations}

ChIP-Seq: chromatin immunoprecipitation sequencing; FDR: false discovery rate; FPKM: fragments per kilobase of transcript per million mapped reads; GTF: gene transfer format; LCAL: lung cancer-associated IncRNA; IncRNA: Iong non-coding RNA; LUAD: lung adenocarcinoma; LUSC: lung squamous cell carcinoma; ORF: open reading frame; qPCR: quantitative real-time PCR; RACE: rapid amplification of CDNA ends; siRNA: short interfering RNA; TCGA: The Cancer Genome Atlas.

\section{Competing interests}

The authors declare that they have no competing interests.

\section{Authors' contributions}

CAM supervised the project. CRC, HXD, and CAM performed computational analyses. NMW and JF-S performed the experimental validation. CRC, NMW, $R G$, and CAM wrote the manuscript. All authors read and approved the final manuscript.

\section{Acknowledgments}

This work was partially funded by a LUNGevity Career Development Award and American Lung Association Biomedical Research Grant (CAM). This work was supported by the National Human Genome Research Institute (NHGRI) U54 HG003079 (PI: Richard K Wilson).

\section{Author details}

${ }^{1}$ Department of Internal Medicine, Division of Oncology, Washington University School of Medicine, St Louis, MO 63110, USA. ${ }^{2}$ The Genome Institute, Washington University School of Medicine, St Louis, MO 63110, USA. ${ }^{3}$ Alvin J Siteman Cancer Center, Washington University School of Medicine, St Louis, MO 63110, USA. Department of Biomedical Engineering, Washington University School of Medicine, St Louis, MO 63110, USA.

Received: 15 April 2014 Accepted: 31 July 2014

Published: 13 August 2014

\section{References}

1. Jemal A, Siegel $R, X u J$, Ward E: Cancer statistics, 2010. CA Cancer J Clin 2010, 60:277-300

2. Prensner JR, Chinnaiyan AM: The emergence of IncRNAs in cancer biology. Cancer Discov 2011, 1:391-407.

3. Gutschner $T$, Diederichs $S$ : The hallmarks of cancer: a long non-coding RNA point of view. RNA Biol 2012, 9:703-719.

4. Derrien $T$, Johnson $R$, Bussotti $G$, Tanzer A, Djebali S, Tilgner H, Guernec G, Martin D, Merkel A, Knowles DG, Lagarde J, Veeravalli L, Ruan X, Ruan Y, Lassmann T, Carninci P, Brown JB, Lipovich L, Gonzalez JM, Thomas M, Davis CA, Shiekhattar R, Gingeras TR, Hubbard TJ, Notredame C, Harrow J, Guigó R: The GENCODE v7 catalog of human long noncoding RNAs: Analysis of their gene structure, evolution, and expression. Genome Res 2012, 22:1775-1789. 
5. Cabili MN, Trapnell C, Goff L, Koziol M, Tazon-Vega B, Regev A, Rinn JL: Integrative annotation of human large intergenic noncoding RNAs reveals global properties and specific subclasses. Genes Dev 2011, 25:1915-1927.

6. Prensner JR, lyer MK, Balbin OA, Dhanasekaran SM, Cao Q, Brenner JC, Laxman B, Asangani IA, Grasso CS, Kominsky HD, Cao X, Jing X, Wang X, Siddiqui J, Wei JT, Robinson D, lyer HK, Palanisamy N, Maher CA, Chinnaiyan AM: Transcriptome sequencing across a prostate cancer cohort identifies PCAT-1, an unannotated lincRNA implicated in disease progression. Nat Biotechnol 2011, 29:742-749.

7. Gupta RA, Shah N, Wang KC, Kim J, Horlings HM, Wong DJ, Tsai M-C, Hung T, Argani P, Rinn JL, Wang Y, Brzoska P, Kong B, Li R, West RB, van de Vijver MJ, Sukumar S, Chang HY: Long non-coding RNA HOTAIR reprograms chromatin state to promote cancer metastasis. Nature 2010, 464:1071-1076.

8. Yu W, Gius D, Onyango P, Muldoon-Jacobs K, Karp J, Feinberg AP, Cui H: Epigenetic silencing of tumour suppressor gene $\mathrm{p} 15$ by its antisense RNA. Nature 2008, 451:202-206.

9. Ji P, Diederichs S, Wang W, Böing S, Metzger R, Schneider PM, Tidow N, Brandt B, Buerger H, Bulk E, Thomas M, Berdel WE, Serve H, Müller-Tidow C: MALAT-1, a novel noncoding RNA, and thymosin beta4 predict metastasis and survival in early-stage non-small cell lung cancer. Oncogene 2003, 22:8031-8041.

10. Rinn JL, Kertesz M, Wang JK, Squazzo SL, Xu X, Brugmann SA, Goodnough LH, Helms JA, Farnham PJ, Segal E, Chang HY: Functional demarcation of active and silent chromatin domains in human HOX loci by noncoding RNAs. Cell 2007, 129:1311-1323.

11. Matouk IJ, DeGroot N, Mezan S, Ayesh S, Abu-lail R, Hochberg A, Galun E: The $\mathrm{H} 19$ non-coding RNA is essential for human tumor growth. PLOS ONE 2007, 2:e845.

12. Kotake Y, Nakagawa T, Kitagawa K, Suzuki S, Liu N, Kitagawa M, Xiong Y: Long non-coding RNA ANRIL is required for the PRC2 recruitment to and silencing of p15(INK4B) tumor suppressor gene. Oncogene 2011, 30:1956-1962.

13. Gutschner T, Hämmerle M, Eissmann M, Hsu J, Kim Y, Hung G, Revenko A, Arun G, Stentrup M, Gross M, Zörnig M, MacLeod AR, Spector DL, Diederichs S: The noncoding RNA MALAT1 is a critical regulator of the metastasis phenotype of lung cancer cells. Cancer Res 2013, 73:1180-1189.

14. Yang F, Huo X, Yuan S, Zhang L, Zhou W, Wang F, Sun S: Repression of the long noncoding RNA-LET by histone deacetylase 3 contributes to hypoxia-mediated metastasis. Mol Cell 2013, 49:1083-1096.

15. Yang Y, Li H, Hou S, Hu B, Liu J, Wang J: The noncoding RNA expression profile and the effect of IncRNA AK126698 on cisplatin resistance in non-small-cell lung cancer cell. PloS One 2013, 8:e65309.

16. Thai $P$, Statt $S$, Chen $C H$, Liang E, Campbell C, Wu R: Characterization of a novel long noncoding RNA, SCAL1, induced by cigarette smoke and elevated in lung cancer cell lines. Am J Respir Cell Mol Biol 2013, 49:204-211.

17. The Cancer Genome Atlas Research Network: Comprehensive genomic characterization of squamous cell lung cancers. Nature 2012, 489:519-525.

18. Seo JS, Ju YS, Lee WC, Shin JY, Lee JK, Bleazard T, Lee J, Jung YJ, Kim JO, Shin JY, Yu SB, Kim J, Lee ER, Kang CH, Park IK, Rhee H, Lee SH, Kim JI, Kang $\mathrm{JH}$, Kim YT: The transcriptional landscape and mutational profile of lung adenocarcinoma. Genome Res 2012, 22:2109-2119.

19. Trapnell C, Williams B, Pertea G, Mortazavi A, Kwan G, van Baren MJ, Salzberg SL, Wold BJ, Pachter L: Transcript assembly and quantification by RNA-Seq reveals unannotated transcripts and isoform switching during cell differentiation. Nat Biotechnol 2010, 28:511-515.

20. Meyer LR, Zweig AS, Hinrichs AS, Karolchik D, Kuhn RM, Wong M, Sloan CA, Rosenbloom KR, Roe G, Rhead B, Raney BJ, Pohl A, Malladi VS, Li CH, Lee BT, Learned K, Kirkup V, Hsu F, Heitner S, Harte RA, Haeussler M, Guruvadoo L, Goldman M, Giardine BM, Fujita PA, Dreszer TR, Diekhans M, Cline MS, Clawson H, Barber GP, et al: The UCSC Genome Browser database: extensions and updates 2013. Nucleic Acids Res 2013, 41:D64-D69.

21. Flicek P, Ahmed I, Amode MR, Barrell D, Beal K, Brent S, Carvalho-Silva D, Clapham P, Coates G, Fairley S, Fitzgerald S, Gil L, García-Girón C, Gordon L, Hourlier T, Hunt S, Juettemann T, Kähäri AK, Keenan S, Komorowska M, Kulesha E, Longden I, Maurel T, McLaren WM, Muffato M, Nag R, Overduin B, Pignatelli M, Pritchard B, Pritchard E, et al: Ensembl 2013. Nucleic Acids Res 2013, 41:D48-D55
22. Harrow J, Frankish A, Gonzalez JM, Tapanari E, Diekhans M, Kokocinski F, Aken BL, Barrell D, Zadissa A, Searle S, Barnes I, Bignell A, Boychenko V, Hunt T, Kay M, Mukherjee G, Rajan J, Despacio-Reyes G, Saunders G, Steward C, Harte R, Lin M, Howald C, Tanzer A, Derrien T, Chrast J, Walters N, Balasubramanian S, Pei B, Tress M, et al: GENCODE: the reference human genome annotation for The ENCODE Project. Genome Res 2012, 22:1760-1774.

23. Pruitt KD, Brown GR, Hiatt SM, Thibaud-Nissen F, Astashyn A, Ermolaeva O, Farrell CM, Hart J, Landrum MJ, McGarvey KM, Murphy MR, O'Leary NA, Pujar S, Rajput B, Rangwala SH, Riddick LD, Shkeda A, Sun H, Tamez P, Tully RE, Wallin C, Webb D, Weber J, Wu W, DiCuccio M, Kitts P, Maglott DR, Murphy TD, Ostell JM: RefSeq: an update on mammalian reference sequences. Nucleic Acids Res 2014, 42:D756-D763.

24. Blanco E, Abril JF: Computational gene annotation in new genome assemblies using GenelD. Methods Mol Biol 2009, 537:243-261.

25. Wang L, Park HJ, Dasari S, Wang S, Kocher JP, Li W: CPAT: Coding-Potential Assessment Tool using an alignment-free logistic regression model. Nucleic Acids Res 2013, 41:e74.

26. Kelley D, Rinn J: Transposable elements reveal a stem cell-specific class of long noncoding RNAs. Genome Biol 2012, 13:R107.

27. Kapusta A, Kronenberg Z, Lynch VJ, Zhuo X, Ramsay L, Bourque G, Yandell M, Feschotte C: Transposable elements are major contributors to the origin, diversification, and regulation of vertebrate long noncoding RNAs. PLoS Genet 2013, 9:e1003470.

28. Van Bakel H, Nislow C, Blencowe BJ, Hughes TR: Most 'dark matter' transcripts are associated with known genes. PLOS Biol 2010, 8:e1000371.

29. Nissan A, Stojadinovic A, Mitrani-Rosenbaum S, Halle D, Grinbaum R, Roistacher M, Bochem A, Dayanc BE, Ritter G, Gomceli I, Bostanci EB, Akoglu $M$, Chen Y-T, Old $\sqcup$, Gure AO: Colon cancer associated transcript-1: a novel RNA expressed in malignant and pre-malignant human tissues. Int J Cancer J Int Cancer 2012, 130:1598-1606.

30. Cao W, Wu W, Shi F, Chen X, Wu L, Yang K, Tian F, Zhu M, Chen G, Wang W, Biddle FG, Gu J: Integrated analysis of long noncoding RNA and coding RNA expression in esophageal squamous cell carcinoma. Int J Genomics 2013, 2013:1-10.

31. Cao W-J, Wu H-L, He B-S, Zhang Y-S, Zhang Z-Y: Analysis of long noncoding RNA expression profiles in gastric cancer. World J Gastroenterol 2013, 19:3658-3664.

32. He W, Cai Q, Sun F, Zhong G, Wang P, Liu H, Luo J, Yu H, Huang J, Lin T: linc-UBC1 physically associates with polycomb repressive complex 2 (PRC2) and acts as a negative prognostic factor for lymph node metastasis and survival in bladder cancer. Biochim Biophys Acta 2013, 1832:1528-1537

33. Wang X-S, Zhang Z, Wang H-C, Cai J-L, Xu Q-W, Li M-Q, Chen Y-C, Qian X-P, Lu T-J, Yu L-Z, Zhang Y, Xin D-Q, Na Y-Q, Chen W-F: Rapid identification of UCA1 as a very sensitive and specific unique marker for human bladder carcinoma. Clin Cancer Res 2006, 12:4851-4858.

34. Li J, Chen Z, Tian L, Zhou C, He MY, Gao Y, Wang S, Zhou F, Shi S, Feng X, Sun N, Liu Z, Skogerboe G, Dong J, Yao R, Zhao Y, Sun J, Zhang B, Yu Y, Shi X, Luo M, Shao K, Li N, Qiu B, Tan F, Chen R, He J: LncRNA profile study reveals a three-IncRNA signature associated with the survival of patients with oesophageal squamous cell carcinoma. Gut 2014, doi:10.1136/gutjhl2013-305806.

35. Lin B, White JT, Ferguson C, Bumgarner R, Friedman C, Trask B, Ellis W, Lange P, Hood L, Nelson PS: PART-1: a novel human prostate-specific, androgen-regulated gene that maps to chromosome $5 q 12$. Cancer Res 2000, 60:858-863.

36. Szafranski P, Dharmadhikari AV, Brosens E, Gurha P, Kolodziejska KE, Zhishuo O, Dittwald P, Majewski T, Mohan KN, Chen B, Person RE, Tibboel D, de Klein A, Pinner J, Chopra M, Malcolm G, Peters G, Arbuckle S, Guiang SF 3rd, Hustead VA, Jessurun J, Hirsch R, Witte DP, Maystadt I, Sebire N, Fisher R, Langston C, Sen P, Stankiewicz P: Small noncoding differentially methylated copy-number variants, including IncRNA genes, cause a lethal lung developmental disorder. Genome Res 2013, 23:23-33.

37. Xi L, Feber A, Gupta V, Wu M, Bergemann AD, Landreneau RJ, Litle VR, Pennathur A, Luketich JD, Godfrey TE: Whole genome exon arrays identify differential expression of alternatively spliced, cancer-related genes in lung cancer. Nucleic Acids Res 2008, 36:6535-6547.

38. Guttman M, Amit I, Garber M, French C, Lin MF, Feldser D, Huarte M, Zuk O, Carey BW, Cassady JP, Cabili MN, Jaenisch R, Mikkelsen TS, Jacks T, Hacohen N, Bernstein BE, Kellis M, Regev A, Rinn JL, Lander ES: Chromatin signature 
reveals over a thousand highly conserved large non-coding RNAs in mammals. Nature 2009, 458:223-227.

39. The Cancer Genome Atlas Research Network: Comprehensive molecular portraits of human breast tumours. Nature 2012, 490:61-70.

40. The Cancer Genome Atlas Research Network: Comprehensive molecular characterization of human colon and rectal cancer. Nature 2012, 487:330-337.

41. The Cancer Genome Atlas Research Network: Comprehensive molecular characterization of clear cell renal cell carcinoma. Nature 2013, 499:43-49.

42. The Cancer Genome Atlas Research Network, Kandoth C, Schultz N, Cherniack AD, Akbani R, Liu Y, Shen H, Robertson AG, Pashtan I, Shen R, Benz CC, Yau C, Laird PW, Ding L, Zhang W, Mills GB, Kucherlapati R, Mardis $E R$, Levine DA: Integrated genomic characterization of endometrial carcinoma. Nature 2013, 497:67-73.

43. Flockhart RJ, Webster DE, Qu K, Mascarenhas N, Kovalski J, Kretz M, Khavari PA: BRAFV600E remodels the melanocyte transcriptome and induces BANCR to regulate melanoma cell migration. Genome Res 2012, 22:1006-1014

44. Kandoth C, McLellan MD, Vandin F, Ye K, Niu B, Lu C, Xie M, Zhang Q, McMichael JF, Wyczalkowski MA, Leiserson MDM, Miller CA, Welch JS, Walter MJ, Wendl MC, Ley TJ, Wilson RK, Raphael BJ, Ding L: Mutational landscape and significance across 12 major cancer types. Nature 2013, 502:333-339.

45. Ohta T, lijima K, Miyamoto M, Nakahara I, Tanaka H, Ohtsuji M, Suzuki T, Kobayashi A, Yokota J, Sakiyama T, Shibata T, Yamamoto M, Hirohashi S: Loss of Keap1 function activates Nrf2 and provides advantages for lung cancer cell growth. Cancer Res 2008, 68:1303-1309.

46. Liu S-P, Yang J-X, Cao D-Y, Shen K: Identification of differentially expressed long non-coding RNAs in human ovarian cancer cells with different metastatic potentials. Cancer Biol Med 2013, 10:138-141.

47. Huang J, Zhou N, Watabe K, Lu Z, Wu F, Xu M, Mo Y-Y: Long non-coding RNA UCA1 promotes breast tumor growth by suppression of p27 (Kip1). Cell Death Dis 2014, 5:e1008.

48. Zhang X-Q, Sun S, Lam K-F, Kiang KM-Y, Pu JK-S, Ho AS-W, Lui W-M, Fung $C-F$, Wong T-S, Leung GK-K: A long non-coding RNA signature in glioblastoma multiforme predicts survival. Neurobiol Dis 2013, 58:123-131.

49. Huarte M, Guttman M, Feldser D, Garber M, Koziol MJ, Kenzelmann-Broz D, Khalil AM, Zuk O, Amit I, Rabani M, Attardi LD, Regev A, Lander ES, Jacks T, Rinn JL: A large intergenic noncoding RNA induced by $\mathrm{p} 53$ mediates global gene repression in the p53 response. Cell 2010, 142:409-419.

50. Trapnell C, Pachter L, Salzberg SL: TopHat: discovering splice junctions with RNA-Seq. Bioinformatics 2009, 25:1105-1111.

51. Quinlan AR, Hall IM: BEDTools: a flexible suite of utilities for comparing genomic features. Bioinformatics 2010, 26:841-842.

52. Robinson MD, Oshlack A: A scaling normalization method for differential expression analysis of RNA-seq data. Genome Biol 2010, 11:R25.

53. Robinson MD, McCarthy DJ, Smyth GK: edgeR: a Bioconductor package for differential expression analysis of digital gene expression data. Bioinformatics 2010, 26:139-140.

54. SigFuge. [http://www.bioconductor.org/packages/devel/bioc/htm//SigFuge.html]

55. Matthews B: Comparison of the predicted and observed secondary structure of T4 phage lysozyme. Biochim Biophys Acta 1975, 405:442-451.

56. UCSC Genome Browser LiftOver. [http://genome.ucsc.edu/cgi-bin/ hgLiftOver]

57. Affymetrix Power Tools. [http://www.affymetrix.com/partners_programs/ programs/developer/tools/powertools.affx]

58. Irizarry RA, Hobbs B, Collin F, Beazer-Barclay YD, Antonellis KJ, Scherf U, Speed TP: Exploration, normalization, and summaries of high density oligonucleotide array probe level data. Biostatistics 2003, 4:249-264.

59. Wang K, Singh D, Zeng Z, Coleman SJ, Huang Y, Savich GL, He X, Mieczkowski P, Grimm SA, Perou CM, MacLeod JN, Chiang DY, Prins JF, Liu J: MapSplice: accurate mapping of RNA-seq reads for splice junction discovery. Nucleic Acids Res 2010, 38:e178.

60. TCGA Pan-cancer MAF files. [http://www.synapse.org/\#!Synapse:syn1729383]
61. Benjamini $Y$, Hochberg $Y$ : Controlling the false discovery rate- a practical and powerful approach to multiple testing. J R Stat Soc Ser B 1995, 57:289-300

62. Kavuri SM, Geserick P, Berg D, Dimitrova DP, Feoktistova M, Siegmund D, Gollnick H, Neumann M, Wajant H, Leverkus M: Cellular FLICE-inhibitory protein (CFLIP) isoforms block CD95- and TRAIL death receptor-induced gene induction irrespective of processing of caspase-8 or cFLIP in the death-inducing signaling complex. J Biol Chem 2011, 286:16631-16646.

doi:10.1186/s13059-014-0429-8

Cite this article as: White et al:: Transcriptome sequencing reveals altered long intergenic non-coding RNAs in lung cancer. Genome Biology 2014 15:429.

\section{Submit your next manuscript to BioMed Central and take full advantage of:}

- Convenient online submission

- Thorough peer review

- No space constraints or color figure charges

- Immediate publication on acceptance

- Inclusion in PubMed, CAS, Scopus and Google Scholar

- Research which is freely available for redistribution

Submit your manuscript at www.biomedcentral.com/submit
C Biomed Central 